\title{
Regulation of Nrf2 Transactivation Domain Activity by p160 RAC3/SRC3 and Other Nuclear Co-Regulators
}

\author{
Wen Lin', Guoxiang Shen', Xiaoling Yuan', Mohit R. Jain ${ }^{1}$, Siwang Yu', \\ Aihua Zhang', J. Don Chen ${ }^{2}$ and Ah-Ng Tony Kong,* \\ ${ }^{1}$ Department of Pharmaceutics, Ernest-Mario School of Pharmacy, Rutgers, The State University of New Jersey, \\ Piscataway, New Jersey 08854, USA \\ ${ }^{2}$ Department of Pharmacology, University of Medicine and Dentistry of New Jersey-Robert Wood Johnson Medical School, \\ Piscataway, New Jersey 08854-5635, USA
}

Received 8 December 2005, Accepted 17 February 2006

Transcription factor NF-E2-related factor 2 (Nrf2) regulates the induction of Phase II detoxifying enzymes and antioxidant enzymes in response to many cancer chemopreventive compounds. In this study, we investigated the role of receptor associated coactivator (RAC3) or steroid receptor coactivator-3 (SRC3) and other nuclear co-regulators including CBP/p300 (CREB-binding protein), CARM1 (Coactivator-associated arginine methyltransferase), PRMT1 (Protein arginine methyl-transferase 1), and p/CAF (p300/ CBP-associated factor) in the transcriptional activation of a chimeric Gal4-Nrf2-Luciferase system containing the transactivation domain (TAD) of Nrf2 in HepG2 cells. The results indicated that $\mathrm{RAC3}$ up-regulated the transactivation activity of Gal4-Nrf2-(1-370) in a dose-dependent manner. The enhancement of transactivation domain activity of Gal4-Nrf2-(1-370) by RAC3 was dampened in the presence of dominant negative mutants of RAC3. Next we studied the effects of other nuclear co-regulators including CBP/ p300, CARM1, PRMT1 and p/CAF, and the results showed that they had different level of positive effects on this transactivation domain activity of Gal4-Nrf2-(1-370). But importantly, synergistic effects of these co-regulators in the presence of $\mathrm{RAC3} / \mathrm{SRC} 3$ on the transactivation activity of Gal4-Nrf2-(1-370) were observed. In summary, our present study showed for the first time that the 160 RAC3/SRC3 is involved in the functional transactivation

\footnotetext{
Abbreviations: Nrf2 (NF-E2-related factor 2), RAC3 (Receptor associated coactivator 3), steroid receptor coactivator-3 (SRC3), CBP/p300 (CREB-binding protein), CARM1 (Coactivator-associated arginine methyltransferase), PRMT1 (Protein arginine methyltransferase 1), p/CAF (p300/CBP-associated factor)
}

*To whom correspondence should be addressed.

Tel: 1-732-445-3831 ext. 228; Fax: 1-732-445-3134

E-mail: kongt@rci.rutgers.edu of TAD of Nrf2 and that the other nuclear co-regulators such as CBP/p300, CARM1, PRMT1 and p/CAF can also transcriptionally activate this TAD of Nrf2 and that they could further enhance the transactivation activity mediated by RAC3/SRC3.

Keywords: CARM1, CBP/p300, Nrf2, p/CAF, PRMT1, RAC3/SRC3

\section{Introduction}

Transcription factor NF-E2-related factor 2 (Nrf2) was demonstrated to regulate the expression of genes encoding antioxidant and phase II detoxifying enzymes, including glutathione S-transferase GST), NADP(H): quinine oxidoreductase (NQO), UDP-glucuronosyltransferase (UGT), heme oxygenase-1 (HO-1) and $\gamma$-glutamylcysteine synthetase ( $\gamma \mathrm{GCS}$ ) (Zhang et al., 1992; Morse and Stoner, 1993; Venugopal and Jaiswal, 1996; Itoh et al., 1997; Chan et al., 2001). As a member of the CNC family of bZIP protein, Nrf2 is mainly sequestered in the cytoplasm by tethering to a cytoskeleton-binding protein (Moi et al., 1994) called Kelch-like erythroid CNC homologue (ECH)-associated protein 1 (Keap1). Upon challenges by oxidative and/or chemical stress induced by reactive oxygen species (ROS), electrophiles as well as many cancer chemopreventive agents, Nrf2 appears to be released from Keap1 binding and enters the nucleus (Itoh et al., 1999; Chan et al., 2001; Nakaso et al., 2003). Upon entering the nucleus, Nrf2 will heterodimerize with small Maf-F/G/K protein, through the leucine zipper dimerization domain and bound to the ARE (antioxidant-response-element) promoter via its DNA binding domain. The ARE has been found to be located in the 5'-flanking region of more than 100 genes than 100 
genes including many antioxidant/phase II enzyme genes (Itoh et al., 1997; Nguyen et al., 2000; Jain et al., 2005). In addition, after $\mathrm{Nrf} 2 / \mathrm{Maf}$ bind to the ARE sequence on target genes, they will also recruit and partner with other nuclear regulatory factors that can modulate (increase or decrease) gene transcription. Recent study indicated that CBP (CREBbinding protein) can bind directly to the Nrf2 transactivation domains Neh4 and Neh5 (Katoh et al., 2001), and that CBP showed synergistic stimulation with Raf on the transactivation activity of Gal4-Nrf 2 (1-370) and full length Nrf2 (Shen et al., 2004).

Gene transcription activation by transcription factors is accomplished through the recruitment of a series of coactivators (Spiegelman and Heinrich, 2004). Coactivator proteins fulfill their functions through protein-protein interactions that bridge transcription factors and basal transcription machinery and through their chromatin remodeling activities that facilitate the assembly of a transcription initiation complex (McManus and Hendzel, 2001; Xu and O'Malley, 2002). The nuclear receptor-associated coactivator-3 (RAC3) or steroid receptor coactivator-3 (SRC3) is a transcriptional coactivator for nuclear receptors and other transcription factors (Li et al., 1997; Belandia and Parker, 2000; Chen et al., 2000; Leo et al., 2000; Soutoglou et al., 2000; Werbajh et al., 2000). RAC3/SRC3 (also known as ACTR,P/CIP and TRAM-1) functions mainly through interaction with transcription factors and recruitment of histone acetylases (HATs) (such as CBP/p300, and p/CAF) and histone methyltransferase (such as CARM-1, PRMT-1) to the promoter for chromatin remodeling and DNA transcription (Chen et al., 1997; Chen et al., 1999; Chen et al., 2000; Stallcup et al., 2000; Demarest et al., 2002; Stallcup et al., 2003; Yadav et al., 2003). Members of SRC family have been shown to enhance gene transactivation by interacting with a wide variety of transcription factors such as serum response factor (Kim et al., 1998), AP-1 (Kim et al., 1997), STAT (Korzus et al., 1997) and NF-кB (Werbajh et al., 2000). We hypothesized that similar to these transcription factors, SRC family protein may also be involved in Nrf-2-mediated transactivation.

In this study, we tested whether RAC3/SRC3 could participate in the stimulation of transactivation activity of Gal4-Nrf 2 (1370) with other coactivators. We found that RAC3/SRC3 alone could enhance the transactivation activity of Gal4-Nrf2(1-370) in a dose-dependant manner. Site-directed mutagenesis on the LXXLL nuclear receptor recognition motif of RAC3/ SRC3 decreased the transactivation activity of Gal4-Nrf2-(1370). Furthermore, we found that CBP, p/CAF, CARM-1 and PRMT1 could further enhance the RAC3/SRC3-mediated transactivation activity on Gal4-Nrf2. Taken together, we showed for the first time that the p160 coactivator RAC3/ SRC3 could stimulate the transactivation activity of Gal4-Nrf 2 (1-370) and this could be further enhanced by the coregulators CBP, p/CAF, CARM-1, and PRMT1.

\section{Material and Methods}

Cell culture. Human hepatoma HepG2 cells were obtained from ATCC (Manassas, VA) and maintained in F-12 medium supplemented with $10 \%$ fetal bovine serum, $1.17 \mathrm{~g} /$ liter of sodium bicarbonate, 0.1 units $/ \mathrm{ml}$ insulin, $0.5 \times$ minimal essential medium amino acid, $100 \mathrm{units} / \mathrm{ml}$ penicillin, and $100 \mu \mathrm{g} / \mathrm{ml}$ streptomycin. Cells were split every 3 days.

Generation of Gal4-Nrf2 chimeras and expression construct. The Gal4-Nrf2 chimera (1-370) was amplified from the Nrf2 template ( 589 amino acids) by polymerase chain reaction using the following primers: 5'-GGTACCTGGATTTGATTGACATACTT-3' (sense) and 5'-TCTAGATTCCAGGGGCACTATCTAGCTCTTC3' (antisense); as described by us previously (Shen et al., 2004). CBP was kindly provided by Robert. H. Goodman (Vollum Institute). CARM1 cDNA was kindly provided by Dr. Michael Stallup, University of Southern California. p/CAF, RAC3/SRC3 and its mutants (the two consecutive leucines within each of the LXXLL motifs in the RAC3/SRC3-ID) was described previously (Li et al., 1997).

Transient transfection and reporter gene activity assays. HepG2 cells were plated in 6-well plates at a density of $4.0 \times 10^{5}$ cells/well. Twenty-four hours after plating, cells were transfected with expression plasmids using LipofectAMINE 2000 (Invitrogen) according to the instruction provided by the manufacturer. In brief, cell culture medium was changed to OPTI-MEM medium before each transfection. Total amount of DNA transfected in each well was adjusted to $4 \mu \mathrm{g}$ by using empty vector pcDNA 3.1. Cells were incubated with transfection mixtures for $5 \mathrm{~h}$ and then cultured in fresh F-12 medium for an additional $36 \mathrm{~h}$ before harvesting. Luciferase activity was determined according to the manufacturer's protocol (Promega). Briefly, cells were washed once with ice-cold phosphate-buffered saline (pH 7.4) and harvested in $1 \times$ Reporter lysis buffer (Promega). After centrifugation, a $20 \mu$ l supernatant was assayed for luciferase activity with a Sirius luminometer (Berthold Detection Systems). The luciferase activity was normalized by protein concentration, determined by $\mathrm{BCA}$ protein assay (Pierce). Data were statistically analyzed by ANOVA, followed by unpaired Student's $t$-test.

\section{Results}

Up-regulation of Gal4-Nrf2(1-370) chimera transactivation activity by RAC3/SRC3. To assess the functional significance of the role of RAC3/SRC3 on Gal4-Nrf2 transcriptional activity, the Gal4-Nrf2 was coexpressed with different doses of RAC3/SRC3 in HepG2 cells. Co-transfection of RAC3/SRC3 resulted in a 1.3-fold, 2.2-fold, 4.1-fold, and 11.8-fold activation of Gal4-Nrf2-(1-370), at doses of $1.0 \mu \mathrm{g}, 2.0 \mu \mathrm{g}, 3.0 \mu \mathrm{g}$ and $4.0 \mu \mathrm{g}$, respectively (Fig. 1A), suggesting that the Gal4-Nrf2-(1-370) transactivation activity increased in a dose-dependent manner by RAC3/SRC3. In order to verify whether RAC3/SRC3 alone increases Gal4-luciferase 

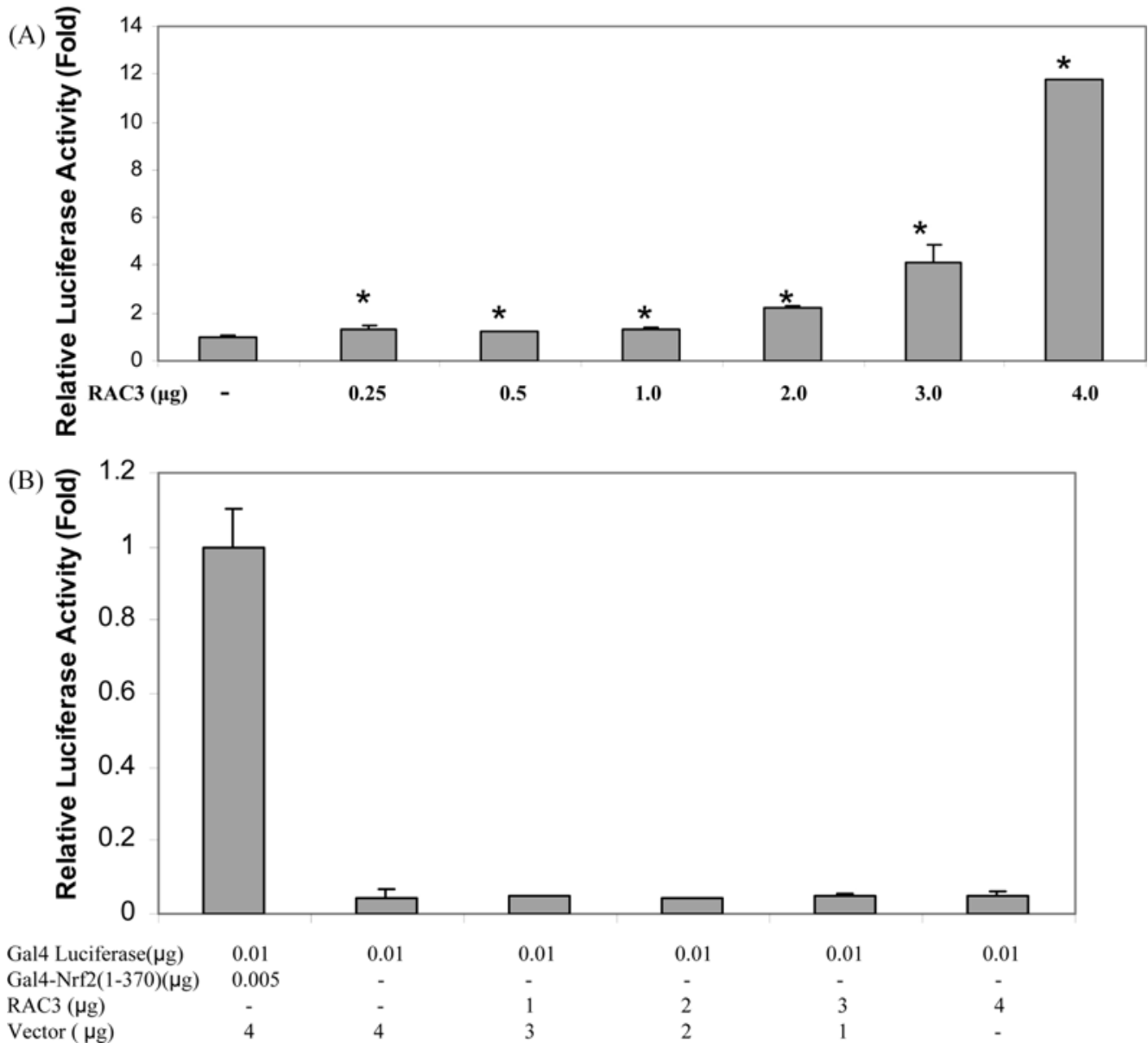

Fig. 1. Regulation of Gal4-Nrf2 chimera transactivation activity by RAC3. HepG2 cells were transiently transferred with 10 ng of Gal4Luc reporter and $5 \mathrm{ng}$ of Gal4-Nrf2- (1-370), together with indicated amounts ( $\mu \mathrm{g}$ ) of RAC3. Empty vector (pcDNA3.1) was used to make up the total DNA to a $4 \mu \mathrm{g}$ /well. Luciferase activity was normalized by protein concentration, determined by BCA protein assay. The luciferase activity of cells transfected with emptor vector was used as control, and its luciferase activity was arbitrarily set as 1 . The data shown are means of three independent experiments performed in duplicate \pm S.D. ${ }^{*}, p<0.05$ versus control.

reporter activity non-specifically, the effect of RAC3/SRC3 on the Gal4-luciferase reporter activity was tested, and the results show that there was no significant changes in the Gal4-luciferase activity, as compared with the control group (Fig. 1B).

Effect of RAC3/SRC3 mutants on the regulation of Gal4-Nrf2 chimera transactivation activity. Within the RAC3/SRC3 coding region, there are six specific motifs sharing a consensus sequence of LXXLL or LLXXL ( $\mathrm{Li}$ et al., 1997). These motifs are required for coactivation function (Leo et al., 2000). RAC3/SRC3 mutant M2 (LL688/9AA) and M3 (LL741/2AA) are located in the receptorinteracting domain (RID). RAC3/SRC3 mutant M4 (DLV1036/7/ 8EAA) has mutation in C-terminal transcriptional activation domain (AD). We compared the effect of wild type RAC3/SRC3 construct with their mutant forms on the induction of Gal4-Nrf2-(1370) transactivation activity. As shown in Fig. 2, at doses of $1.0 \mu \mathrm{g}$ and $2.0 \mu \mathrm{g}$, there were no significant differences between their effects on the Gal4-Nrf2-(1-370) transactivation activity. When RAC3/SRC3 and its mutants were overexpressed at $4.0 \mu \mathrm{g}$, all the RAC3/SRC3 mutants significantly attenuated Gal4-Nrf2-(1-370) transactivation activity (Fig. 2), suggesting the specificity of this activation. This specificity was further confirmed by our coimmunoprecipitation experiments showing that the full lengths of Nrf2 and RAC3/SRC3 could be co-immunoprecipitated (unpublished observations).

Enhancement of RAC3/SRC3-mediated regulation of Gal4Nrf2 chimera transactivation activity by $\mathbf{C B P}$ and p/CAF. Next we tested whether the histone acetylation proteins, CBP or $\mathrm{p} / \mathrm{CAF}$ had any activity on Gal4-Nrf2-(1-370), and found that a 2.0-and a 2.4-fold increase of RAC3/SRC3-mediated transactivation, respectively in HepG2 cells (Fig. 3). In addition, we investigated whether CBP and $\mathrm{p} / \mathrm{CAF}$ could induce RAC3/SRC3 mediated Gal4-Nrf2 activation in these cells. Cotransfection of both CBP and RAC 3 induced Gal4-Nrf2-(1-370) transactivation activity about 5.7-fold. Cotransfection of both p/CAF and RAC3/SRC3 enhanced Gal4Nrf2-(1-370) transactivation activity to about 7.8-fold (Fig. 3). These results suggested that $\mathrm{CBP}$ and $\mathrm{p} / \mathrm{CAF}$ could enhance with RAC 3 to induce Gal4-Nrf2-(1-370) transactivation activity in HepG2 cells. 


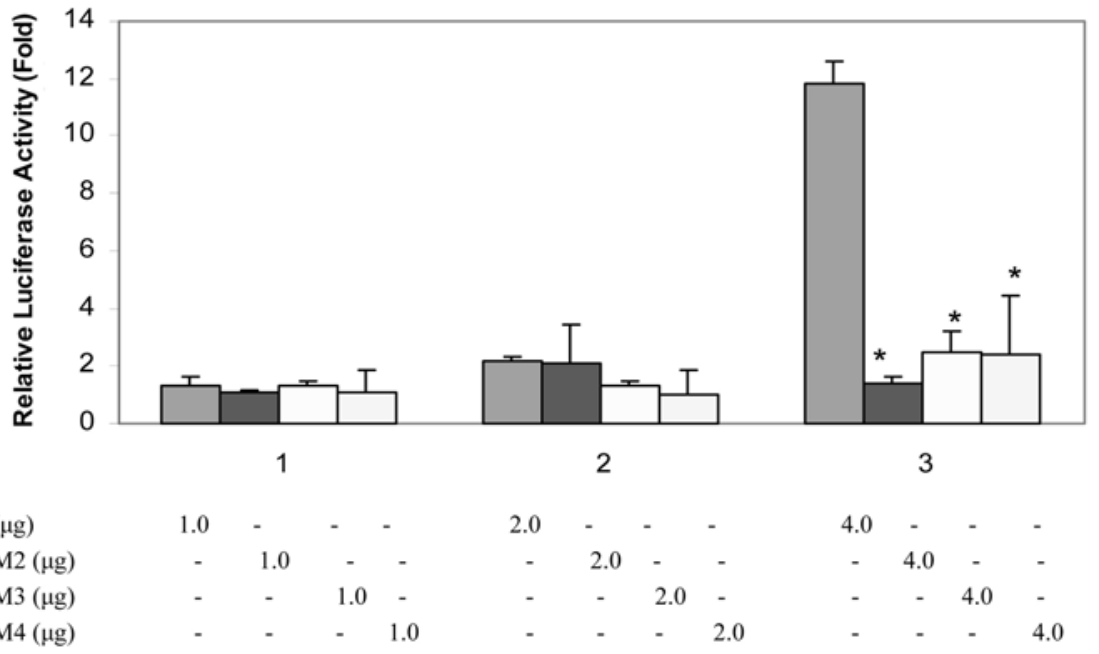

Fig. 2. Regulation of Gal4-Nrf2 chimera transactivation activity by RAC3 and its mutants. HepG2 cells were transiently transfected with $10 \mathrm{ng}$ of Gal4-Luc reporter and $5 \mathrm{ng}$ of Gal4-Nrf2- (1-370), together with indicated amounts ( $\mu \mathrm{g})$ of SRC-3. Empty vector (pcDNA3.1) was used to make up the total DNA to a $4 \mu \mathrm{g} /$ well. Luciferase activity was normalized by protein concentration, determined by BCA protein assay. The luciferase activity of cells transfected with RAC3 vector was used as control. The data shown are means of three independent experiments performed in duplicate \pm S.D. ${ }^{*}, p<0.05$ versus control.

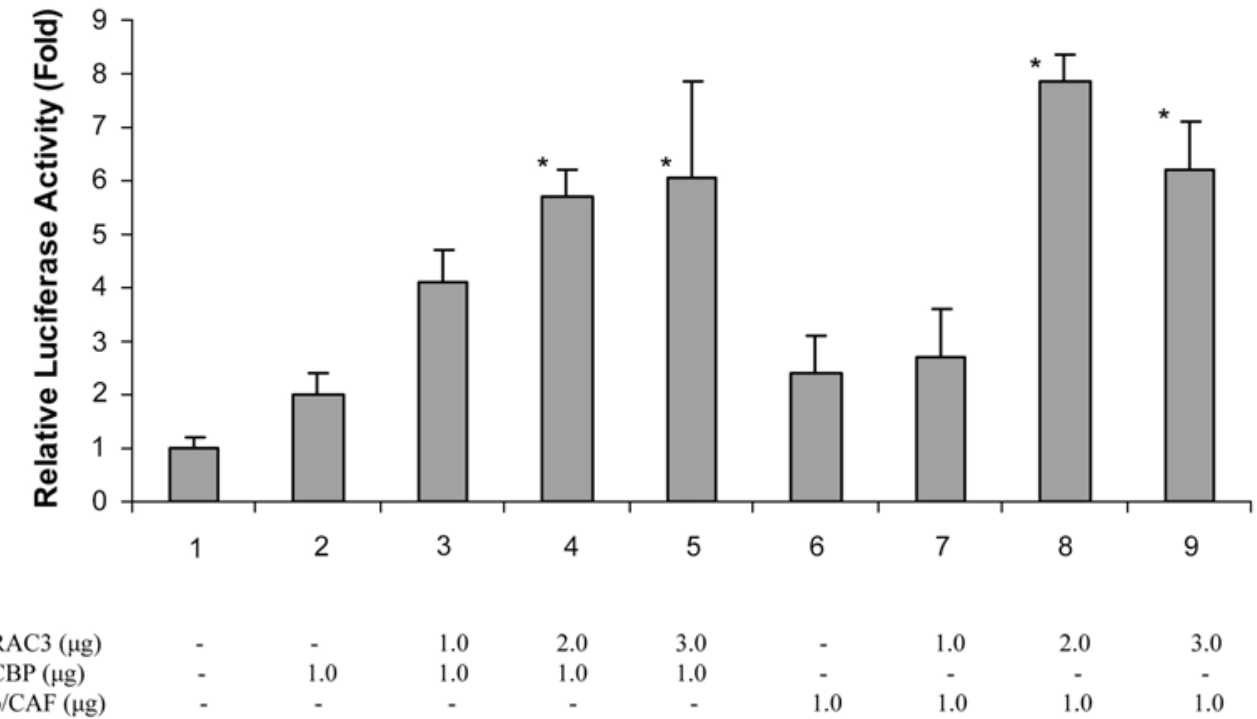

Fig. 3. Regulation of Gal4-Nrf2 chimera transactivation activity by RAC3, CBP and p/CAF. HepG2 cells were transiently transferred with $10 \mathrm{ng}$ of Gal4-Luc reporter and $5 \mathrm{ng}$ of Gal4-Nrf2- (1-370), together with indicated amounts ( $\mu \mathrm{g}$ ) of SRC-3, CBP and p/CAF. Empty vector (pcDNA3.1) was used to make up the total DNA to a $4 \mu \mathrm{g} /$ well. Luciferase activity was normalized by protein concentration, determined by BCA protein assay. The luciferase activity of cells transfected with emptor vector was used as control, and its luciferase activity was arbitrarily set as 1 . The data shown are means of three independent experiments performed in duplicate \pm S.D.*, $p<0.05$ versus CBP $1.0 \mu \mathrm{g}$ or $\mathrm{p} / \mathrm{CAF} 1.0 \mu \mathrm{g}$.

Enhancement of RAC3/SRC3-mediated regulation of Gal4Nrf2 chimera transactivation activity by CARM-1, PRMT1. CARM1 belongs to the PRMT family of arginine-specific protein methyltransferases, which share a 330 amino acid-region that contains the methyltransferase activity (Lin et al., 1996; Chen et al., 1999). Once bound to the promoter, CARM1 exerts its effect through methylation of histones and enhances protein-protein interactions (Teyssier et al., 2002). PRMT1 is the predominant member of family of protein arginine methyltransferases (PRMT).
We found that both CARM1 and PRMT1 induced the transactivation activity of Gal4-Nrf2-(1-370) (Fig. 4). Cotransfection of CARM1 and RAC3/SRC3 further enhanced the Gal4-Nrf2-(1-370) transactivation activity significantly. Similar effect was also observed with the coexpression of PRMT1 and RAC3/SRC3 on Gal4-Nrf2 (1-370) transactivation activity in HepG2 cells (Fig. 4), which suggested that a potential synergistic stimulation of CARM1 and PRMT1 on RAC3/SRC3-mediated Gal4-Nrf2-(1-370) transactivation activity. 


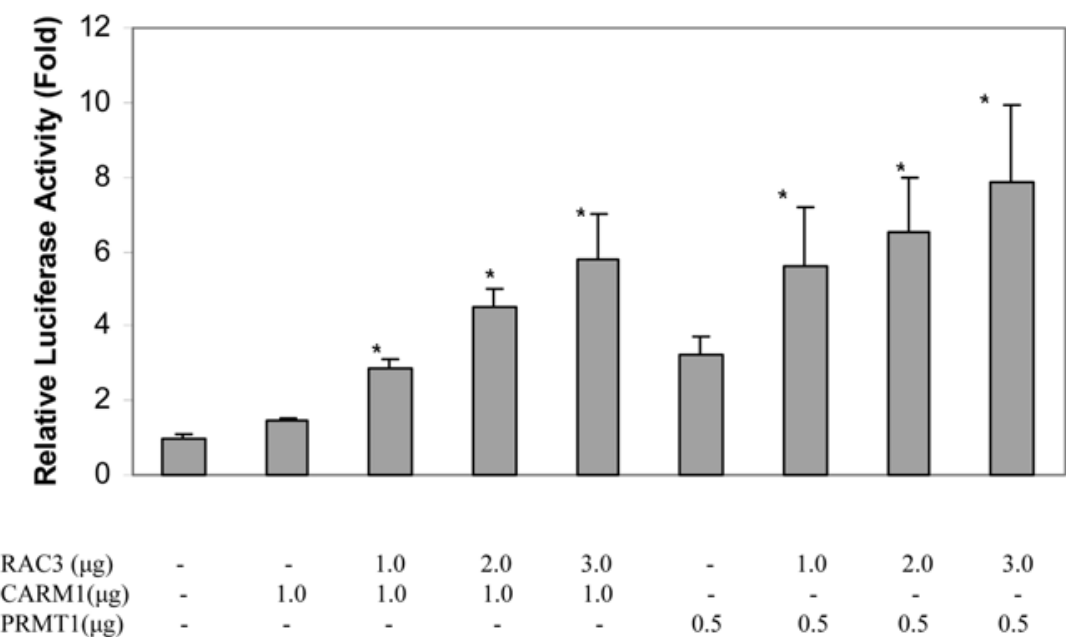

Fig. 4. Regulation of Gal4-Nrf2 chimera transactivation activity by RAC3, CARM1 and PRMT1. HepG2 cells were transiently transfected with $10 \mathrm{ng}$ of Gal4-Luc reporter and $5 \mathrm{ng}$ of Gal4-Nrf2- (1-370), together with indicated amounts ( $\mu \mathrm{g})$ of RAC3, CARM1 and PRMT1. Empty vector (pcDNA3.1) was used to make up the total DNA to a $4 \mu \mathrm{g} /$ well. Luciferase activity was normalized by protein concentration, determined by BCA protein assay. The luciferase activity of cells transfected with empty vector was used as control, and its luciferase activity was arbitrarily set as 1 . The data shown are means of three independent experiments performed in duplicate \pm S.D. $*, p<0.05$ versus control.

\section{Discussion}

$\mathrm{Nrf} 2$ is an important transcription regulator of ARE-mediated gene expression of antioxidant and phase II detoxifying enzymes in response to a variety of stimuli including ROS, oxidants, electrophiles, xenobiotics and many dietary cancer chemopreventive compounds. Once Nrf2 enters the nucleus, the mechanism by which $\mathrm{Nrf} 2$ recruits the transcriptional machinery remains unknown. Our present study shows that the nuclear receptor coactivator RAC3/SRC3 enhances the transactivation activity of the chimeric Gal4-Nrf2-(1-370). The Gal4-Nrf2-(1-370) construct, which contains the Nrf2 transactivation domain (TAD), has very high basal transactivation activity, similar to to the full-length Nrf2's transcriptional activity on the ARE (Shen, Hebbar et al., 2004). We also show that the histone acetylation related co-activators $\mathrm{CBP}$ and $\mathrm{p} /$ $\mathrm{CAF}$, and histone methylation related co-activators CARM-1 and PRMT-1 could further enhance the transactivation activity of Gal4-Nrf2 (1-370) mediated by RAC3/SRC3, suggesting their positive roles in this system.

The p160 coactivators contribute to transcriptional activation by bringing other associated coregulatory proteins to the promoter. The 160 coactivator complex includes either of the two related proteins $\mathrm{p} 300$ or $\mathrm{CBP}$, which bind to the activation domain-1 (AD-1) of p160 coactivators and function as coactivators for many DNA-binding transcriptional activators (Li et al., 1997). CBP and p300 contribute to chromatin remodeling by acetylating histones, and also acetylate other components of the transcription initiation complex (Chen et al., 1999). CBP and p300 can also bind directly to the basal transcription factors and may thereby facilitate the assembly of the transcription initiation complex (Chen et al., 1997).
Along with acetylation of histones by $\mathrm{CBP} / \mathrm{p} 300$, methylation of histone $\mathrm{H} 3$ and possibly other proteins in the transcription initiation complex by CARM1 could also be important to help remodel the chromatin structure and recruit RNA polymerase II. CARM1 methylates histone H3 and PRMT1 methylates histone H4 (Stallcup et al., 2000; Ma et al., 2001; An et al., 2004). We found that CARM1 or PRMT1 cotransfected with Gal4-Nrf2 slightly increased Gal4-Nrf2 (1-370) transactivation activity, while coexpression of RAC3/SRC-3 and CARM1 significantly stimulated Gal4-Nrf2 (1-370) transactivation activity.

From the above results, it could be postulated that Nrf2 would be able to recruit the basic transcriptional control machinery to efficiently transcribe the target genes, and these include the various co-activators such as the p300 family $[\mathrm{CBP} / \mathrm{p} 300 / \mathrm{pCAF}]$, the p160 family of co-activators [SRC1/ TIF2/RAC3], as well as the CARM1/PRMT1 proteins, as described previously by Rosenfeld and Glass (Rosenfeld and Glass, 2001). Future studies focusing on the biochemical, molecular and functional interactions between these proteins complexes will be needed to arrive at a more physiologically relevant model of Nrf2-mediated transcription of critical genes including cancer chemopreventive genes as well as genes involved in inflammation, cardiovascular diseases and central nervous system. In summary, our current study shows for the first time that the p160 coactivator RAC3/SRC3 could stimulate the transactivation activity of Gal4-Nrf 2 (1-370) and that this transactivation activity could be further enhanced by the coregulators such as CBP, p/CAF, CARM-1 and PRMT1.

Acknowledgments We thank Drs. Michael Stallcup, University of Southern California and Dr. Robert H. Goodman (Vollum 
Institute, Oregon Health Sciences University) for providing the reagents and the members of Dr. Kong's lab for their assistance and critical discussion on this manuscript. This work was supported in part by NIH grants R01-CA-94828 (to A.-N.T.K.).

\section{References}

An, W., Kim, J. and Roeder, R. G. (2004) Ordered cooperative functions of PRMT1, p300, and CARM1 in transcriptional activation by $\mathrm{p} 53$. Cell 117, 735-748.

Belandia, B. and Parker, M. G. (2000) Functional interaction between the p160 coactivator proteins and the transcriptional enhancer factor family of transcription factors. J. Biol. Chem. 275, 30801-30805.

Chan, K., Han, X. D. and Kan, Y. W. (2001) An important function of $\mathrm{Nrf} 2$ in combating oxidative stress: detoxification of acetaminophen. Proc. Natl. Acad. Sci. USA 98, 4611-4616.

Chen, D., Huang, S. M. and Stallcup, M. R. (2000) Synergistic, p160 coactivator-dependent enhancement of estrogen receptor function by CARM1 and p300. J. Biol. Chem. 275, 4081040816.

Chen, D., Ma, H., Hong, H., Koh, S. S., Huang, S. M., Schurter, B. T. Aswad, D. W. and Stallcup, M. R. (1999) Regulation of transcription by a protein methyltransferase. Science 284, 21742177.

Chen, H., Lin, R. J., R. J. Lin, Schiltz, R. L., Chakravarti, D., Nash, A., Nagy, L., Privalsky, M. L, Nakatani, Y. and Evans, R. M. (1997) Nuclear receptor coactivator ACTR is a novel histone acetyltransferase and forms a multimeric activation complex with P/CAF and CBP/p300. Cell 90, 569-580.

Chen, H., Lin, R. J., Xie, W. Wilpitz, D. and Evans, R. M. (1999) Regulation of hormone-induced histone hyperacetylation and gene activation via acetylation of an acetylase. Cell 98, 675686.

Demarest, S. J., Martinez-Yamout, M., Chung, J. Chen, H., Xu, W. Dyson, H. J. Evans, R. M. and Wright, P. E. (2002) Mutual synergistic folding in recruitment of $\mathrm{CBP} / \mathrm{p} 300$ by p160 nuclear receptor coactivators. Nature 415, 549-553.

Itoh, K., Chiba, T., Takahashi, S., Ishii, T., Igarashi, K., Katoh, Y., Oyake, T., Hayahshi, N., Satoh, K., Hatayama, I., Yamamoto, M. and Nabeshima, Y. (1997) An Nrf2/small Maf heterodimer mediates the induction of phase II detoxifying enzyme genes through antioxidant response elements. Biochem. Biophys. Res. Commun. 236, 313-322.

Itoh, K., Wakabayashi, N., Katoh, Y., Ishii, T., Igarashi, K., Engel, J. D. and Yamamoto, M. (1999) Keap1 represses nuclear activation of antioxidant responsive elements by Nrf2 through binding to the amino-terminal Neh2 domain. Genes Dev. 13, 76-86.

Jain, A. K., Bloom, D. A. and Jaiswal, A. K. (2005) Nuclear import and export signals in control of NRF2. J. Biol. Chem. 280, 29158-29168.

Katoh, H., Itoh, S., Shen, J. R. and Ikeuchi, M. (2001) Functional analysis of psbV and a novel c-type cytochrome gene psbV2 of the thermophilic cyanobacterium Thermosynechococcus elongatus strain BP-1. Plant Cell Physiol. 42, 599-607.

Kim, H., Pennie, W. D., Sun, Y. and Colburn, N. H. (1997)
Differential functional significance of AP-1 binding sites in the promoter of the gene encoding mouse tissue inhibitor of metalloproteinases-3. Biochem. J. 324, 547-553.

Kim, H. J., Kim, J. H. and Lee, J. W. (1998) Steroid receptor coactivator-1 interacts with serum response factor and coactivates serum response element-mediated transactivations. J. Biol. Chem. 273, 28564-28567.

Korzus, E., Nagase, H., Rydell, R. and Travis, J. (1997) The mitogen-activated protein kinase and JAK-STAT signaling pathways are required for an oncostatin M-responsive elementmediated activation of matrix metalloproteinase 1 gene expression. J. Biol. Chem. 272, 1188-1196.

Leo, C., Li, H. and Chen, J. D. (2000) Differential mechanisms of nuclear receptor regulation by receptor-associated coactivator 3 . J. Biol. Chem. 275, 5976-5982.

Li, H., Gomes, P. J. and Chen, J. D. (1997) RAC3, a steroid/ nuclear receptor-associated coactivator that is related to SRC-1 and TIF2. Proc. Natl. Acad. Sci. USA 94, 8479-8484.

Lin, W. J., Gary, J. D., Yang, M. C., Clarke, S. and Herschman, H. R. (1996) The mammalian immediate-early TIS 21 protein and the leukemia-associated BTG1 protein interact with a protein-arginine N-methyltransferase. J. Biol. Chem. 271, 15034-15044.

Ma, H., Baumann, C. T., Li, H., Strahl, B. D., Rice, R., Jelinek, M. A., Aswad, D. W., Allis, C. D., Hager, G. L. and Stallcup, M. R. (2001) Hormone-dependent, CARM1-directed, argininespecific methylation of histone $\mathrm{H} 3$ on a steroid-regulated promoter. Curr. Biol. 11, 1981-1985.

McManus, K. J. and M. J. Hendzel (2001) CBP, a transcriptional coactivator and acetyltransferase. Biochem. Cell Biol. 79, 253266.

Moi, P., Chan, K. and Asunis, I., Cao, A. and Kan Y. W. (1994) Isolation of NF-E2-related factor 2 (Nrf2), a NF-E2-like basic leucine zipper transcriptional activator that binds to the tandem NF-E2/AP1 repeat of the beta-globin locus control region. Proc. Natl. Acad. Sci. USA 91, 9926-9930.

Morse, M. A. and G. D. Stoner (1993) Cancer chemoprevention: principles and prospects. Carcinogenesis 14, 1737-1746.

Nakaso, K., Yano, H., Fukuhara, Y. Takeshima, T., Wada-Isoe, K. and Nakashima, K. (2003) PI3K is a key molecule in the Nrf2mediated regulation of antioxidative proteins by hemin in human neuroblastoma cells. FEBS Lett 546, 181-184.

Nguyen, T., Huang, H. C. and Pickett, C. B. (2000) Transcriptional regulation of the antioxidant response element. Activation by Nrf2 and repression by MafK. J. Biol. Chem. 275, 15466-15473.

Rosenfeld, M. G. and Glass, C. K. (2001) Coregulator codes of transcriptional regulation by nuclear receptors. J. Biol. Chem. 276, 36865-36868.

Shen, G., Hebbar, V. Nair, S., Xu, C., Li, W. Lin, W., Keum, Y. S. Han, J. Gallo, M. A. and Tony Kong, A.-N. (2004) Regulation of Nrf2 transactivation domain activity. The differential effects of mitogen-activated protein kinase cascades and synergistic stimulatory effect of Raf and CREB-binding protein. J. Biol. Chem. 279, 23052-23060.

Soutoglou, E., Papafotiou, G., Katrakili, N. and Talianidis, I. (2000) Transcriptional activation by hepatocyte nuclear factor-1 requires synergism between multiple coactivator proteins. $J$. Biol. Chem. 275, 12515-12520.

Spiegelman, B. M. and Heinrich, R. (2004) Biological control 
through regulated transcriptional coactivators. Cell 119, 157167.

Stallcup, M. R., Chen, D., Koh, S. S., Ma, H., Lee, Y.-H, Li, H., Schurter, B. T. and Aswad, D. W. (2000) Co-operation between protein-acetylating and protein-methylating co-activators in transcriptional activation. Biochem. Soc. Trans. 28, 415-418.

Stallcup, M. R., Kim, J. H., Teyssier, C., Lee, Y. H., Ma, H. and Chen, D. (2003) The roles of protein-protein interactions and protein methylation in transcriptional activation by nuclear receptors and their coactivators. J. Steroid Biochem. Mol. Biol. 85, 139-145.

Teyssier, C., Chen, D. and Stallcup, M. R. (2002) Requirement for multiple domains of the protein arginine methyltransferase CARM1 in its transcriptional coactivator function. J. Biol. Chem. 277, 46066-46072.

Venugopal, R. and A. K. Jaiswal (1996) Nrf1 and Nrf2 positively and c-Fos and Fral negatively regulate the human antioxidant response element-mediated expression of $\mathrm{NAD}(\mathrm{P}) \mathrm{H}:$ quinone oxidoreductase 1 gene. Proc. Natl. Acad. Sci USA 93, 1496014965.

Werbajh, S., I. Nojek, et al. (2000) RAC-3 is a NF-kappa B coactivator. FEBS Lett 485, 195-199.

$\mathrm{Xu}$, J. and O'Malley, B. W. (2002) Molecular mechanisms and cellular biology of the steroid receptor coactivator (SRC) family in steroid receptor function. Rev. Endocr. Metab. Disord. 3, 185-192.

Yadav, N., J. Lee, et al. (2003) Specific protein methylation defects and gene expression perturbations in coactivatorassociated arginine methyltransferase 1-deficient mice. Proc. Natl. Acad. Sci USA 100, 6464-6468.

Zhang, Y., P. Talalay, et al. (1992) A major inducer of anticarcinogenic protective enzymes from broccoli: isolation and elucidation of structure. Proc. Natl. Acad. Sci USA 89, 2399-2403. 